\title{
Differential effect of quetiapine and lithium on functional connectivity of the striatum in first episode mania
}

Orwa Dandash ${ }^{1,2}$, Murat Yücel ${ }^{1,2}$, Rothanthi Daglas ${ }^{3,4}$, Christos Pantelis $\mathbb{E}^{2,5}$, Patrick McGorry ${ }^{3,4}$, Michael Berk $\mathbb{B}^{3,4,6,7,8}$ and Alex Fornito ${ }^{1}$

\begin{abstract}
Mood disturbances seen in first-episode mania (FEM) are linked to disturbed functional connectivity of the striatum. Lithium and quetiapine are effective treatments for mania but their neurobiological effects remain largely unknown. We conducted a single-blinded randomized controlled maintenance trial in 61 FEM patients and 30 healthy controls. Patients were stabilized for a minimum of 2 weeks on lithium plus quetiapine then randomly assigned to either lithium (serum level $0.6 \mathrm{mmol} / \mathrm{L}$ ) or quetiapine (dosed up to $800 \mathrm{mg} /$ day) treatment for 12 months. Resting-state fMRI was acquired at baseline, 3 months (patient only) and 12 months. The effects of treatment group, time and their interaction, on striatal functional connectivity were assessed using voxel-wise general linear modelling. At baseline, FEM patients showed reduced connectivity in the dorsal $(p=0.05)$ and caudal $(p=0.008)$ cortico-striatal systems when compared to healthy controls at baseline. FEM patients also showed increased connectivity in a circuit linking the ventral striatum with the medial orbitofrontal cortex, cerebellum and thalamus $(p=0.02)$. Longitudinally, we found a significant interaction between time and treatment group, such that lithium was more rapid, compared to quetiapine, in normalizing abnormally increased functional connectivity, as assessed at 3-month and 12-month followups. The results suggest that FEM is associated with reduced connectivity in dorsal and caudal corticostriatal systems, as well as increased functional connectivity of ventral striatal systems. Lithium appears to act more rapidly than quetiapine in normalizing hyperconnectivity of the ventral striatum with the cerebellum. The study was registered on the Australian and New Zealand Clinical Trials Registry (ACTRN12607000639426). http://www.anzctr.org.au
\end{abstract}

\section{Introduction}

First-episode mania (FEM) patients can exhibit difficulties in emotion regulation and deficits in cognitive function such as attention, response inhibition and working memory ${ }^{1}$ that are commonly linked to the functional integrity of neural circuits connecting prefrontal cortex (PFC) with subcortical and limbic structures $^{2-5}$.

\footnotetext{
Correspondence: Orwa Dandash (orwa.dandash@unimelb.edu.au)

${ }^{1}$ Brain \& Mental Health Laboratory, School of Psychological Sciences \& Monash Institute of Cognitive and Clinical Neurosciences, Monash University, Clayton, VIC 3168, Australia

${ }^{2}$ Melbourne Neuropsychiatry Centre, Department of Psychiatry, The University of Melbourne and Melbourne Health, Carlton South, VIC, Australia
}

Full list of author information is available at the end of the article
Key among these are the cortico-striato-thalamic (CST) and cortico-striato-cerebellar (CSC) networks, which are thought to mediate a broad array of cognitive, motor and affective processes ${ }^{6,7}$. The striatum is as an important structure in these networks, representing a key point for integrating diverse cortical and cerebellar inputs. Broadly, the functional organization of the striatum evolves along dorsal-to-ventral and medial-to-lateral axes which separate the associative striatum (head of the caudate nucleus and dorsorostral putamen) from the motor (caudate tail and dorsocaudal putamen) and the limbic (ventral striatum/nucleus accumbens) subdivisions ${ }^{6,8}$. The striatum is thus well-positioned to play a critical role in functions that integrate cognitive processes with emotional drives to 
implement motor outputs-an ability that is thought to be fundamentally disrupted in bipolar disorder ${ }^{9}$.

A popular method for studying striatal and other brain networks is to use functional magnetic resonance imaging (fMRI) to record coordinated, spontaneous brain dynamics in the absence of an explicit task (the so-called resting-state) ${ }^{10,11}$. The signal fluctuations recorded with resting-state fMRI are organized into well-defined networks $^{12}$, including specific CST and CSC systems ${ }^{13-15}$. These networks are robust over time $\mathrm{e}^{16,17}$, heritable ${ }^{18}$ and influence task-evoked activity and behavior ${ }^{19,20}$, suggesting that they represent an intrinsic and functionally important property of brain activity ${ }^{21}$.

Several resting-state fMRI studies have found that patients with bipolar disorder show disturbances in coordinated brain activity-so-called functional connectivity-between the striatum and other brain $\operatorname{areas}^{22-24}$. In the largest resting-state study to date, increased connectivity between a medial paralimbic resting-state network, involving the ventromedial prefrontal cortex and the ventral striatum, with a network thought to subserve emotion regulation and executive function, including subgenual cingulate and insula, distinguished bipolar patients from schizophrenia patients and healthy controls, suggesting that interactions between cognitive and emotional networks may be specifically disrupted in bipolar disorder ${ }^{24}$. Similarly, another analysis found that functional connectivity between the basal ganglia, thalamus and cortical areas implicated in cognition were the most distinguishing feature between bipolar patients and healthy controls, achieving a classification accuracy of $90 \%{ }^{25}$. Furthermore, altered functional connectivity in the cognitive and affective subdivisions of the striatum has been shown to differentiate manic patients from depressed bipolar patients ${ }^{26}$. Collectively, these findings suggest that bipolar disorder is associated with abnormal coupling between striatal and other brain areas involved in cognitive and emotional processes.

Most fMRI studies have included patients treated with the mood stabilizer lithium, second-generation antipsychotics such as quetiapine, or adjunctive therapy ${ }^{22-24}$. The evidence from randomized-controlled studies suggests that quetiapine is an effective treatment of bipolar mania $^{27}$ and bipolar depression ${ }^{28}$, for maintenance therapy $^{29}$, and as an adjunct treatment to lithium, where efficacy may be greater than quetiapine monotherapy ${ }^{30}$. However, no study to date has investigated the differential effect of monotherapy of either of the drugs on restingstate functional connectivity in First Episode Mania (FEM) patients. This is mainly due to the low incidence of FEM and the consequent difficulty in recruiting affected patients $^{31,32}$. In addition, previous studies have used cross-sectional designs, which do not allow an assessment of how treatment changes brain function over time. More importantly, there are no randomized designs in these cohorts.

In this study, we aimed to investigate changes in the resting-state functional connectivity of four key regions of the striatum, encompassing dorsal (cognitive), ventral (affective) and caudal (motor) circuits in FEM patients randomized to either quetiapine or lithium. Patients were scanned at baseline, 3 months and 12 months, with a sample of healthy controls also scanned at the baseline and 12-month assessments. We expected that functional connectivity of both the dorsal and ventral striatum would be altered in patients compared to controls at baseline. We also predicted, in light of the evidence for the clinical efficacy of both lithium and quetiapine in treating manic patients $^{27,29,30}$, that treatment with both drugs would normalize baseline abnormalities over the follow-up period.

\section{Subjects and methods \\ Participants}

Sixty-one participants with FEM were recruited via Orygen Youth Health and Monash Health between December 2008 and December 2013. Patients were screened with the Structured Clinical Interview for DSMIV-TR by trained clinicians and were diagnosed with mania (bipolar I disorder, schizoaffective disorder, or a substance-induced mood disorder). Individuals presenting with an acute manic episode with psychotic features and who had not been previously treated for a manic episode were stabilised on a combination of quetiapine plus lithium in an open label manner as part of a routine care protocol. Following provision of informed oral and written consent, patients were randomised after remission (2-3 months), to lithium or quetiapine monotherapy. Patients were required to have been on a combination of quetiapine and lithium as standard therapy for at least 1 month prior to randomisation, and to have a score of at least 20 on the Young Mania Rating Scale (YMRS) ${ }^{33}$ during their acute manic episode. Female patients were required to be using effective contraception if they were sexually active and of childbearing age. Patients of 15-25 years of age, who were fluent in English, had the capacity to provide informed consent, and comply with study procedures, were randomised to treatment allocation of either lithium or quetiapine monotherapy. The Melbourne Health and Monash Health Human Research Ethics Committees approved the protocol in accordance with the Helsinki Declaration.

\section{Exclusion criteria}

Patients were excluded for known or suspected clinically relevant systemic medical disorder, organic mental disease or a history of epilepsy; sensitivity or allergy to quetiapine, lithium or any additives in the medication; 
prior use of medication with a cytochrome P450 3A4 inhibiting effect in the 14 days preceding enrolment; inability to comply with the requirements of giving informed consent or the treatment protocol; immediate risk of self-harm or of harming others; pregnancy or breastfeeding; or diabetes mellitus.

\section{Randomisation and blinding}

At the discretion of the treating team an independent statistician generated a computerised randomisation sequence 2-3 months following stabilisation from a FEM. A randomisation $\log$ was established and a set of sequentially ordered envelopes was kept in a locked filing cabinet at the Orygen Research Centre. The patients, treating psychiatrist and case managers knew which treatment the patient was receiving while research assistants, neuropsychologists, and all individuals involved in neuroimaging, analysis, and data management remained blinded to this information.

\section{Study protocol}

Of the 61 recruited subjects, 31 subjects were allocated to quetiapine treatment and 30 patients were allocated to lithium treatment. A single researcher (RD) conducted all clinical and diagnostic assessments. Clinical assessments were carried out at baseline and on fortnightly intervals for the first month, then on a monthly basis for the following 2 months, and then at 3 monthly intervals thereafter concluding at the 1-month time point. These included observer-based ratings using the $\mathrm{YMRS}^{33}$ for manic symptoms, the Montgomery-Åsberg Depression Rating Scale MADRS; ${ }^{34}$ for depressive symptoms, the Brief Psychiatric Rating Scale BPRS; ${ }^{35}$; for psychotic symptoms, and the Clinical Global Impressions scale for use in Bipolar disorder CGI-BP; ${ }^{36}$; to determine overall symptom severity. Control subjects underwent MRI scanning at baseline and at the 12-month time points whereas FEM patients were scanned at baseline, 3 and 12month time points to assess response to treatment.

\section{MRI data acquisition}

A single scanner at the Murdoch Children's Research Institute at Royal Children's Hospital in Melbourne, Australia (3 T Siemens Trio Tim, 32 channel head coil) was used to acquire high-resolution structural T1 Magnetization-Prepared RApid Gradient-Echo $(\text { MPRAGE) })^{37}$ scans for each subject. Image acquisition parameters at every time-point were as follows: 192 sagittal slices with a nominal $1 \mathrm{~mm}^{3}$ voxel size, $256 \mathrm{~mm} \times 232 \mathrm{~mm}$ Field-of-View (FoV) and a matrix size of $256 \times 192$ or $512 \times 384$ pixel resolution (the latter had $0.5 \times 0.5 \mathrm{~mm}$ in-plane resolution), $2000 \mathrm{~ms}$ repetition time (TR) and $2.24 \mathrm{~ms}$ echo time (TE). T2*-weighted echoplanar images were acquired under eyes-closed resting- state conditions. Subsequent participant debriefing ensured that no participants fell asleep during the scan. Functional MRI was acquired using a T2*-weighted sequence with $3.3 \times 3.3 \times 3.0 \mathrm{~mm}$ voxel size, a TR of $2400 \mathrm{~ms}$, TE of $30 \mathrm{~ms}$, flip angle of $90^{\circ}$ in $64 \times 64$ matrix size and $384 \mathrm{~mm}$ FoV. A total of 307 volumes comprising 36 slices each were acquired.

\section{Imaging preprocessing}

An established procedure was used to characterize corticostriatal functional connectivity in relation to four seed regions located in ventral and dorsal areas of the caudate nucleus and putamen per hemisphere ${ }^{13,38,39}$. Seeds were defined in both hemispheres as $3.5 \mathrm{~mm}$ radial spheres at the following stereotaxic coordinates: dorsal caudate $(x= \pm 13, y=15, z=9)$; ventral striatum/nucleus accumbens $(x= \pm 9, y=9, z=-8)$; dorsal-caudal putamen $(x= \pm 28, y=1, z=3)$ ventral-rostral putamen $(x=$ $\pm 20, y=12, z=-3$ ). A secondary seed-based analysis was conducted in which two $3.5 \mathrm{~mm}$ spherical seeds were placed in the right and left visual cortex as described elsewhere $^{40}$. Image preprocessing was performed using statistical parametric mapping software (SPM8) and included slice-timing correction, motion correction via affine transformation to the first image and coregistration of functional images with subjects' anatomical scans, which were concurrently normalized to the SPM-T1 template. The resulting transformation matrix was applied to the functional data to achieve accurate spatial normalization across individuals. The anatomical scans were also segmented using a unified normalization and segmentation approach ${ }^{41}$.

White matter and CSF were generated by thresholding the corresponding tissue images segmented from the $\mathrm{T} 1$ scan at 99 and $50 \%$ tissue probability, respectively. Any overlap with the grey matter mask (thresholded at 1\%) was removed by image subtraction. Voxelwise time series were extracted from the white matter and CSF masks and subjected to the aCompCor method ${ }^{42}$, in which voxelwise time course from each tissue mask were subjected to separate principal component analyses. The first five components from each mask were retained as nuisance regressors modelling physiological noise and head motion effects. All data were linearly detrended and a linear regression model that included these ten component signals, together with the six head motion parameters (three rotation, three translation) estimated during the head motion correction procedure, and the first-order derivatives of all sixteen signals were then fitted on a voxelwise basis. This approach has been shown to successfully control for head motion and physiological noise in fMRI data ${ }^{43}$. The noise-corrected data were then band pass filtered $(0.008<f<0.08)$ and spatially smoothed with a Gaussian filter ( $8 \mathrm{~mm}$ full-width at half-maximum). All 
image sequences were routinely inspected for potential normalization artifacts. Mean time series were extracted from each striatal seed in both hemispheres. The data of one patient at baseline was excluded due to excessive head motion ( $>3 \mathrm{~mm}$ translation and $>3^{\circ}$ rotation). This resulted in a final sample of 30 control subjects, and 40 patients at baseline. The final sample for both treatment groups at 3 months (denoted $\mathrm{m} 3$ ) was 13 subjects in the quetiapine group and 16 subjects in the lithium group. At 12 months (denoted $\mathrm{m} 12$ ), there was 20 subjects in the control group, 12 subjects in the quetiapine group and 14 subjects in the lithium group.

\section{First-level, within-subjects analysis}

For each participant, functional connectivity maps were estimated using general linear models (GLM) as implemented in SPM8. Time courses extracted from each striatal sub-region were entered into a single GLM that included all striatal seeds as covariates of interest in a whole-brain regression analysis. Contrast images were generated for each participant by estimating the regression coefficient between all brain voxels and each region's time-series for left and right hemisphere seeds.

\section{Second-level, between-condition analysis}

Separate second-level models were used to test for (1) baseline disease-related changes in corticostriatal effects, and (2) the longitudinal effects of treatment. For the first analysis, separate models were used to test for group differences in the functional connectivity of each of the four seed regions, with group (patient, control) and hemisphere (left and right) as covariates of interest and age and gender as nuisance covariates. Between-group statistical maps were thresholded at a $P<.001$ uncorrected and subjected to cluster-based correction for multiple comparison at $P<.05$ determined by a Monte Carlo simulation test ${ }^{44}$ based on the recently amended 3dClustSim algorithm ${ }^{45}$.

The longitudinal effect of treatment was characterized using a $3 \times 2$ random-effects flexible factorial design with group (control, quetiapine and lithium) and time (baseline, $\mathrm{m} 12$ ) as factors and age and gender as nuisance covariates. A separate $2 \times 3$ random-effect design was used to characterize the effect of treatment at $\mathrm{m} 3$ in the patients groups only. Longitudinal effects were mapped by masking the $F$-contrast for the group $\times$ time interaction with the statistical map of the baseline differences between patients and controls to ensure that the influence of treatment was characterized in brain regions that are (1) functionally connected to the striatum; and (2) of primary pathophysiological relevance to the disorder. Interaction effects within this mask that survived a threshold of $P<.05$ small volume-corrected, were deemed significant.

\section{Clinical associations}

Change from baseline scores were computed for the BPRS total ( $\triangle$ BPRStot12), MADRS ( $\triangle$ MADRS12), CGIBP severity of depression ( $\triangle$ CGI-BPsevDepres12), and CGI-BP overall severity of bipolar ( $\triangle$ CGI-BPsevBP12) by subtracting $\mathrm{m} 12$ clinical scores from baseline scores ${ }^{46}$.

\section{Results}

\section{Demographics and clinical outcomes}

Of the 61 recruited subjects, 11 in the quetiapine treatment group and 9 in the lithium treatment group were excluded at or before randomization took place for the following reasons; relapse at baseline or prior to commencement of monotherapy $(n=5)$; self-ceasing of all medications $(n=3)$; non-stability on monotherapy ( $n$ $=2)$; preference for the non-randomized medication $(n=$ $3)$; clinician withdrawal due to side effects $(n=2)$ and treatment disengagement $(n=4)$ (Fig. 1). Twenty-one patients were allocated to quetiapine treatment and 20 subjects were allocated to lithium. Two additional subjects were excluded at baseline; one subject due to never being on monotherapy and one subject due to noncompliance to randomized medication (see CONSORT flowchart in Supplement for more information) rendering a final sample of 19 subjects in the quetiapine group and 20 subjects in the lithium group. Three patients in the quetiapine group and four in the lithium group discontinued after their baseline participation.

Treatment groups were matched for age, gender, handedness and IQ but only matched for age and handedness with the control group (Table 1). Baseline clinical scores did not differ between the two treatment groups (patients assigned to lithium or quetiapine), indicating that stabilization was successful. As reported in refs 46,47, the lithium group demonstrated significant improvement compared to quetiapine after 12 months on rating scales of depression, psychosis, and mood instability.

\section{Baseline effects of disease}

At baseline, all patients (i.e., both treatment groups), when compared to controls, demonstrated increased functional connectivity in the putative affective corticostriatal network, linking the ventral striatum (nucleus accumbens) and the ventromedial orbitofrontal cortex (vmOFC), cerebellum, and the thalamus in the right hemisphere (Fig. 1a). Patients showed reduced functional connectivity between the dorsal caudate and DLPFC in the right hemisphere (Fig. 1; Table 2). Patients also showed reduced connectivity between the dorsocaudal putamen and the premotor area, and between the same putamen seed and the caudate nucleus bilaterally (Fig. 1; Table 2). There was no significant difference in functional connectivity between the two treatment groups at baseline. 


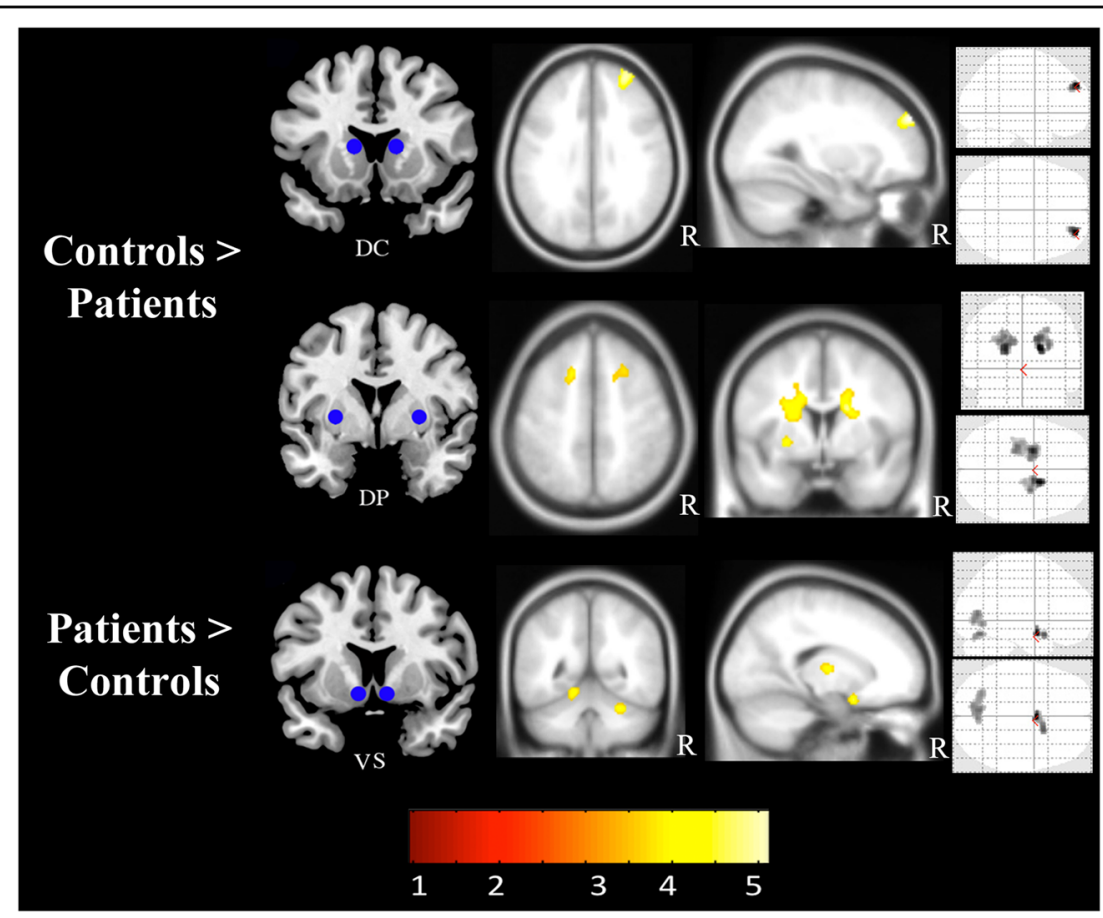

Fig. 1 Z-score statistical map of significant baseline comparison in functional connectivity between control subjects and first-episode mania patients collapsed across both treatment groups; quetiapine and lithium. DC dorsal caudate nucleus, DP dorsal putamen, VS ventral striatum including the nucleus accumbens. R right hemisphere. Insets show results overlaid on glass brain. Results are displayed at $P<0.05$ (FWE cluster corrected). For more information see Supplementary Materials

Table 1 Demographics of treatment groups and control subjects

\begin{tabular}{|c|c|c|c|c|c|c|c|c|}
\hline \multirow[t]{2}{*}{ Demographics } & \multicolumn{2}{|c|}{ Control $N=\mathbf{3 0}$} & \multicolumn{2}{|c|}{ Quetiapine $(N=19)$} & \multicolumn{2}{|c|}{ Lithium $(N=20)$} & \multicolumn{2}{|c|}{ Statistics } \\
\hline & $N$ & $\%$ & $N$ & $\%$ & $N$ & $\%$ & $x^{2}$ & $P$ \\
\hline \multicolumn{9}{|l|}{ Gender } \\
\hline Male & 12 & 40 & 14 & 70 & 16 & 84 & 9.87 & .007 \\
\hline Female & 18 & 60 & 5 & 30 & 4 & 16 & & \\
\hline \multicolumn{9}{|l|}{ Handedness $^{\mathrm{a}}$} \\
\hline Right-handed & 26 & 93 & 15 & 83 & 19 & 95 & 3.64 & .162 \\
\hline Left-handed & 2 & 7 & 3 & 17 & 0 & 0 & & \\
\hline \multirow[t]{2}{*}{ Age } & Mean & s.d. & Mean & s.d. & Mean & s.d. & $F$ & P \\
\hline & 21.40 & 2.46 & 21.47 & 2.14 & 21.45 & 2.31 & 0.145 & .88 \\
\hline Premorbid IQ (WTAR) & 105.43 & 10.82 & 92.89 & 13.80 & 96.71 & 13.89 & 11.37 & .001 \\
\hline \multicolumn{9}{|l|}{ Diagnosis } \\
\hline Bipolar disorder & - & - & 19 & 100 & 17 & 85 & 1.89 & 1.17 \\
\hline Schizoaffective disorder & - & - & 1 & 5 & 1 & 5 & 0.04 & 1.84 \\
\hline Substance-induced Mania & - & - & 1 & 5 & 1 & 5 & 0.08 & 1.80 \\
\hline
\end{tabular}

${ }^{a}$ Data for two subjects in the control group and one subject in the quetiapine and the lithium group were missing.

bWTAR: Wechsler Test of Adult Reading (UK scaled score) 
Table 2 Brain regions demonstrating significant between-group differences in functional connectivity $(P<0.05$; FWE cluster corrected) and group $\times$ time interactions $(P<0.05$; small-volume corrected)

\begin{tabular}{|c|c|c|c|c|c|}
\hline Main Effect & Anatomical Region & Hemisphere & MNI Peak Coordinates $(x, y, z)$ & Z-score & Voxels \\
\hline DC & DLPFC & Right & $30,54,34$ & 4.16 & 156 \\
\hline \multirow[t]{5}{*}{ PTdc } & Caudate Nucleus & Right & $18,6,22$ & 4.53 & 632 \\
\hline & Putamen Globus Pallidus & Left & $-26,-2,-2$ & 4.28 & 167 \\
\hline & Caudate Nucleus & Left & $-22,-2,20$ & 4.27 & 1043 \\
\hline & Premotor Cortex & Left & $-18,12,56$ & 3.56 & 183 \\
\hline & Premotor Cortex & Right & $24,14,46$ & 3.52 & 120 \\
\hline \multirow[t]{4}{*}{ VS } & mOFC & Right & $4,0,-18$ & 4.59 & 225 \\
\hline & Thalamus & Right & $16,-12,6$ & 3.94 & 140 \\
\hline & Cerebellum & Left & $-14,-48,-14$ & 3.93 & 120 \\
\hline & Cerebellum & Right & $28,-50,-28$ & 3.91 & 134 \\
\hline \multicolumn{6}{|c|}{ Group x Time Interaction } \\
\hline VS & Cerebellum & Right & $32,-48,-28$ & 3.73 & 73 \\
\hline
\end{tabular}

DC dorsal caudate, PTdc dorsocaudal Putamen, VS ventral striatum.

\section{Longitudinal effects of treatment}

A significant group $\times$ time interaction was identified for functional connectivity between the ventral striatum and right cerebellum (Fig. 2 and Table 2). Post-hoc $t$-tests showed that both treatment groups showed increased connectivity when compared to control subjects at baseline (quetiapine $t_{1,68}=3.0, P=.001$ cluster-corrected; lithium $t_{1,68}=3.6, P=.001$ cluster-corrected). At $\mathrm{m} 3$, patients treated with lithium showed reduced functional connectivity compared to those treated with quetiapine $\left(t_{1,27}=2.0, P<.05 \mathrm{svc}\right)$. After $\mathrm{m} 12$, the two treatment groups did not differ in their connectivity strength, although both were significantly higher than controls (Fig. 2; quetiapine $t_{1,30}=1.6, \quad P<.05 \quad \mathrm{svc}$; lithium $\left.t_{1,34}=1.75, P<.05 \mathrm{svc}\right)$.

\section{Clinical associations}

In an exploratory analysis, we next examined whether the treatment-related changes in functional connectivity were related to symptom improvement. We extracted estimate of functional connectivity from the cerebellar region showing a significant group $\times$ time interaction and correlated these estimates with measures of symptom change. In the lithium group, functional connectivity between the ventral striatum and cerebellum was associated with symptom improvement on the following scales in the lithium group: $\triangle$ CGI-BPsevDepres12 $(r=$ $0.51, n=14, P=.022)$ and $\Delta$ CGI-BPsevBP12 $(r=0.51, n$ $=14, P=.021)$. However, none of these associations survived full Bonferroni correction for multiple comparisons $(\alpha=0.0125)$.

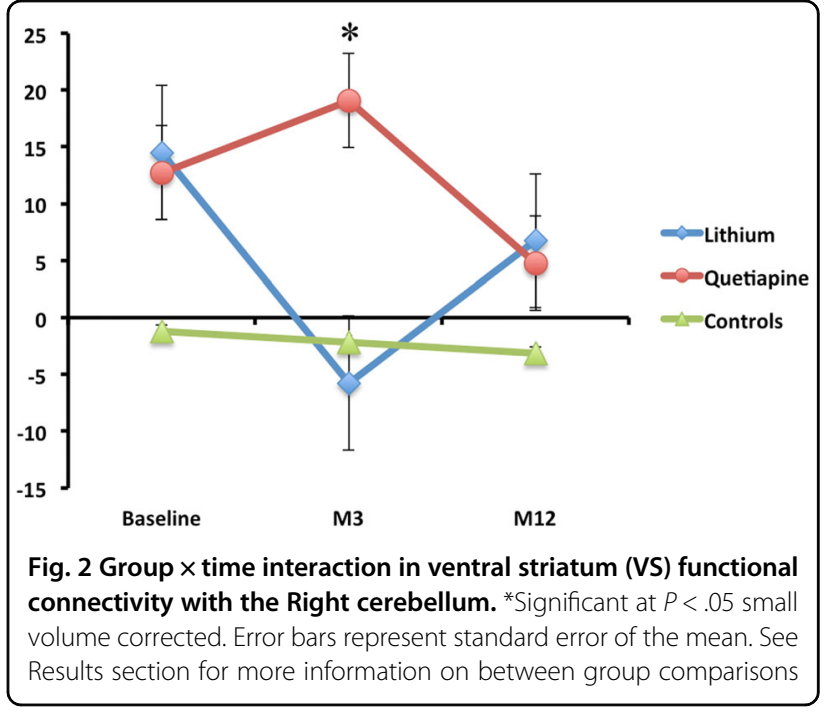

An additional exploratory association analysis was conducted in which baseline functional connectivity estimates, outside of the brain regions that represent between-group differences (Fig. 1), were examined to see whether they predict symptom improvement after 12 months. Symptom scores were entered as variables of interest in a voxel-wise whole-brain regression analysis while controlling for age and gender. Baseline functional connectivity predicted improvement in symptom scores in both treatment groups after 12 months (Supplementary Figures 2 and 3). Specifically, connectivity of the ventral striatum with the right temporal cortex predicted improvement on BPRS $(r=0.83, n=14, P=.012$ FWE 
Cluster corrected) while connectivity between the ventral striatum and the right middle frontal gyrus predicted improvement on MADRS scores $(r=0.86, n=14, P$ $=.001$ FWE Cluster corrected) in the lithium group (Supplementary Figure 1). In the quetiapine group, connectivity of the ventral striatum and the ACC predicted improvement on BPRS scores $(r=0.68, n=12, P=.001$ FWE Cluster corrected) (Supplementary Figure 2). These effects survived full Bonferroni correction for multiple comparisons $(\alpha=0.0125)$.

There was no clinical association with the connectivity of the visual cortex as suggested by the secondary analysis (Supplementary Figure 1). This latter analysis supports the specificity of the results to ventral striatum connectivity.

\section{Discussion}

FEM patients showed reduced functional connectivity of dorsal cortico-striatal circuitry, coupled with abnormally increased functional connectivity of a ventral CSC network. The connectivity abnormalities normalized to a similar extent over the 12-month period of this study in patients treated with either lithium or quetiapine, but the changes occurred earlier in the lithium-treated group, being apparent at 3 months. These findings suggest that the therapeutic effect of quetiapine in FEM may be delayed relative to lithium, at least with respect to normalizing functional connectivity of the ventral striatum with the cerebellum. We note however, that the abnormalities did not completely normalize, with residual differences between the two patient groups and healthy controls still apparent at 12 months.

Paralleling its traditional role in controlling movement, the cerebellum plays an important role in modulating emotions ${ }^{48,49}$. The cerebellum sends monosynaptic projections to the basal ganglia as well as other limbic subcortical structures including the hippocampus and the amygdala ${ }^{7,48}$. Lesions to the posterior and vermal cerebellum lead to disinhibition and inappropriate behavior, a characteristic feature of mania ${ }^{50}$. Moreover, the cerebellum commonly appears as one of the most activated brain regions during emotion appraisal ${ }^{51,52}$ and a reduction in cerebellar volume is associated with the number of affective episodes, including manic and hypomanic episodes, in bipolar patients ${ }^{53}$.

In a recent volumetric study of this cohort, we found reduced cerebellar volume in FEM patients at baseline, when compared to control subjects, in the same region that shows increased connectivity with the ventral striatum $^{54}$. These findings further support the role of the cerebellum in mediating affect and suggest that increased functional connectivity in the limbic circuit may be related to the structural deficit in the cerebellum. One hypothesis is that increased functional connectivity with the ventral striatum represents a compensatory response to a structural lesion in this area, although testing this hypothesis would require a thorough behavioural assessment $^{55}$. Unlike our previous study, which found that cerebellar volume was not responsive to treatment ${ }^{54}$, we report here that medication improves striatocerebellar connectivity, suggesting that functional measures may be more sensitive to the effects of pharmacotherapy (Fig. 2).

Changes in functional connectivity of the ventral striatum were accompanied by improvement in symptoms after 12 months in the lithium group only. This finding was further cemented by the secondary exploratory analysis in which baseline functional connectivity of the ventral striatum predicted improvement in depressive and general psychiatric symptoms in the lithium group but only general psychiatric symptoms in the quetiapine group (Supplementary Figures 2-3). As noted in another study of this sample ${ }^{46}$, the findings favour lithium over quetiapine and point to a recurrent theme that implicates the ventral striatum as a major culprit in mediating manic symptoms and potentially the pharmacological effect of treatment.

The increased ventral striatal connectivity with the vmOFC and medial thalamus observed in patients at baseline is another indicator that the canonical ventral (limbic) corticostriatal circuit is compromised in mania. The vmOFC coordinates and integrates somatosensory information, including emotion, in decision-making and plays a role in assigning positive value to stimuli ${ }^{56}$. Bipolar patients and their unaffected first degree relatives illicit similar responses in the vmOFC even in the absence of rewarding stimuli, and have previously been shown to exhibit alterations in striatothalamic functional connectivity akin to their affected relatives ${ }^{24,57}$. These findings suggest that functional connectivity disturbances in ventral striatum connectivity could be a trait marker for the illness. This notion is further supported by the observation that increased connectivity of the ventral striatum was present at baseline in both treatment groups after the stabilization phase. In other words, the disturbance may not be related to the onset of the illness per se but to other biological factors closer to the etiology of the illness ${ }^{58}$.

Notably, FEM patients also showed reduced functional connectivity in the dorsal corticostriatal system at baseline. We have observed a similar gradient of dorsal-toventral, hypoconnectivity-to-hyperconnectivity in a separate sample of patients with first episode schizophrenialike psychosis and their unaffected relatives ${ }^{38}$. We have also found hypoconnectivity of the dorsal system in people with an at-risk mental state for psychosis ${ }^{59}$. Most patients in this study had experienced an episode of psychosis, suggesting that dorsal corticostriatal hypoconnectivity may be a risk marker for psychosis that 
transcends traditional diagnostic boundaries. Further work examining how corticostriatal connectivity covaries with specific symptoms or syndromes across diagnoses will be required to test this hypothesis.

Strengths of the study include the relative homogeneity of the sample, the selection of a first episode cohort, randomized assignment to treatment groups and a longitudinal design. The study included $10 \%$ of individuals with schizoaffective disorder and substance-induced mania, which may have influenced the results, potentially favoring quetiapine given its efficacy in treating schizoaffective disorders ${ }^{60}$. Nonetheless, the number of subjects with either disorder was matched in the two treatment groups, thus reducing the variability contributed by these diagnoses and rendering the study more representative of FEM patient samples in Western societies $^{22,24}$. Limitations include the relatively modest sample, which is expected for such a difficult to recruit and engage population. Multi-site investigations may prove useful in establishing the samples required for more robust multivariate methods to assess the utility of striatal functional connectivity in predicting treatment effects.

\begin{abstract}
Acknowledgements
This study was supported by an unrestricted grant from Astra Zeneca. The funding organization had no role in the design and conduct of the study; collection, management, analysis, and interpretation of the data; preparation, review, or approval of the manuscript; and decision to submit the manuscript for publication. MB was supported by a NHMRC Senior Principal Research Fellowship 1059660. C.P. was supported by a NHMRC Senior Principal Research Fellowship (ID: 628386 \& 1105825). P.M. was supported by a NHMRC SPR fellowship. A.F. was supported by the Australian Research Council (ID: FT130100589) and NHMRC (ID: 1050504). M.Y. was supported by a National Health and Medical Research Council Fellowship (ID: 1117188) and the David Winston Turner Endowment Fund.
\end{abstract}

\section{Author details}

'Brain \& Mental Health Laboratory, School of Psychological Sciences \& Monash Institute of Cognitive and Clinical Neurosciences, Monash University, Clayton, VIC 3168, Australia. ${ }^{2}$ Melbourne Neuropsychiatry Centre, Department of Psychiatry, The University of Melbourne and Melbourne Health, Carlton South, VIC, Australia. ${ }^{3}$ Orygen, The National Centre of Excellence in Youth Mental Health, 35 Poplar Road, Parkville, VIC 3052, Australia. ${ }^{4}$ Centre for Youth Mental Health, University of Melbourne, 35 Poplar Road, Parkville, VIC 3052, Australia. ${ }^{5}$ Florey Institute for Neuroscience and Mental Health, Kenneth Myer Building, Royal Parade, Parkville VIC Australia. ${ }^{6}$ IMPACT Strategic Research Centre, School of Medicine, Deakin University, Geelong VIC 3220, Australia. ${ }^{7}$ Orygen Youth Health Clinical Program, 35 Poplar Road, Parkville VIC 3052, Australia. ${ }^{8}$ Barwon Health and the Geelong Clinic, Swanston Centre, Geelong, VIC 3220, Australia

\section{Conflict of interest}

M.B. has received Grant/Research Support from Bristol Myers Squibb, Eli Lilly, Glaxo SmithKline, Meat and Livestock Board, Organon, Novartis, Mayne Pharma, Servier and Woolworths, has been a speaker for Astra Zeneca, Bristol Myers Squibb, Eli Lilly, Glaxo SmithKline, Janssen Cilag, Lundbeck, Merck, Pfizer, Sanofi Synthelabo, Servier, Solvay and Wyeth, and served as a consultant to Astra Zeneca, Bioadvantex, Bristol Myers Squibb, Eli Lilly, Glaxo SmithKline, Janssen Cilag, Lundbeck Merck and Servier. C.P. has participated on Advisory Boards for Janssen-Cilag, Astra-Zeneca, Lundbeck, and Servier and has received honoraria for talks presented at educational meetings organised by AstraZeneca, Janssen-Cilag, Eli-Lilly, Pfizer, Lundbeck and Shire. P.M. has received investigator initiated research grants from Astra Zeneca, Janssen Cilag, Eli Lilly, and BMS. He has received honoraria for educational events from Janssen Cilag, Eli Lilly, BMS, Astra Zeneca, Pfizer and Lundbeck.

\section{Publisher's note}

Springer Nature remains neutral with regard to jurisdictional claims in published maps and institutional affiliations.

Supplementary Information accompanies this paper at (https://doi.org/ 10.1038/s41398-018-0108-8).

Received: 15 April 2017 Revised: 28 October 2017 Accepted: 30 December 2017

Published online: 06 March 2018

\section{References}

1. Daglas, R. et al. Cognitive impairment in first-episode mania: a systematic review of the evidence in the acute and remission phases of the illness. Int. J. Bipolar Disord. 3, 9 (2015).

2. Vargas, C., Lopez-Jaramillo, C. \& Vieta, E. A systematic literature review of resting state network--functional MRI in bipolar disorder. J. Affect. Disord. 150, 727-735 (2013).

3. Marchand, W. R. \& Yurgelun-Todd, D. Striatal structure and function in mood disorders: a comprehensive review. Bipolar Disord. 12, 764-785 (2010).

4. Price, J. L., Carmichael, S. T. \& Drevets, W. C. Networks related to the orbital and medial prefrontal cortex; a substrate for emotional behavior? Prog. Brain. Res. 107, 523-536 (1996).

5. Price, J. L. \& Drevets, W. C. Neural circuits underlying the pathophysiology of mood disorders. Trends Cogn. Sci. 16, 61-71 (2012).

6. Alexander, G. E., DeLong, M. R. \& Strick, P. L. Parallel organization of functionally segregated circuits linking basal ganglia and cortex. Annu. Rev. Neurosci. 9, 357-381 (1986).

7. Bostan, A. C., Dum, R. P. \& Strick, P. L. The basal ganglia communicate with the cerebellum. Proc. Natl. Acad. Sci. USA 107, 8452-8456 (2010).

8. Haber, S. N. The primate basal ganglia: parallel and integrative networks. J. Chem. Neuroanat. 26, 317-330 (2003).

9. McKenna, B. S. \& Eyler, L. T. Overlapping prefrontal systems involved in cognitive and emotional processing in euthymic bipolar disorder and following sleep deprivation: a review of functional neuroimaging studies. Clin. Psychol. Rev. 32, 650-663 (2012).

10. Biswal, B., Yetkin, F. Z., Haughton, V. M. \& Hyde, J. S. Functional connectivity in the motor cortex of resting human brain using echo-planar MRI. Magn. Reson. Med. 34, 537-541 (1995).

11. Fornito, A. \& Bullmore, E. T. What can spontaneous fluctuations of the blood oxygenation-level-dependent signal tell us about psychiatric disorders? Curr. Opin. Psychiatr. 23, 239-249 (2010).

12. Smith, S. M. et al. Correspondence of the brain's functional architecture during activation and rest. Proc. Natl. Acad. Sci. USA 106, 13040-13045 (2009).

13. Di Martino, A. et al. Functional connectivity of human striatum: a resting state FMRI study. Cereb. Cortex. 18, 2735-2747 (2008).

14. Harrison, B. J. et al. Altered corticostriatal functional connectivity in obsessivecompulsive disorder. Arch. Gen. Psychiatry 66, 1189-1200 (2009).

15. Bernard, J. A. et al. Resting state cortico-cerebellar functional connectivity networks: a comparison of anatomical and self-organizing map approaches. Front. Neuroanat. 6, 31 (2012).

16. Damoiseaux, J. S. et al. Consistent resting-state networks across healthy subjects. Proc. Natl. Acad. Sci. USA 103, 13848-13853 (2006).

17. Shehzad, Z. et al. The resting brain: unconstrained yet reliable. Cereb. Cortex. 19, 2209-2229 (2009).

18. Fornito, A. et al. Genetic influences on cost-efficient organization of human cortical functional networks. J. Neurosci. 31, 3261-3270 (2011).

19. Fox, M. D., Snyder, A. Z., Vincent, J. L. \& Raichle, M. E. Intrinsic fluctuations within cortical systems account for intertrial variability in human behavior. Neuron $\mathbf{5 6}$, 171-184 (2007)

20. Fox, M. D., Snyder, A. Z., Zacks, J. M. \& Raichle, M. E. Coherent spontaneous activity accounts for trial-to-trial variability in human evoked brain responses. Nat. Neurosci. 9, 23-25 (2006).

21. Fox, M. D. \& Raichle, M. E. Spontaneous fluctuations in brain activity observed with functional magnetic resonance imaging. Nat. Rev. Neurosci. 8, 700-711 (2007).

22. Anticevic, A. et al. Archival report: global prefrontal and fronto-amygdala dysconnectivity in bipolar I disorder with psychosis history. Biol. Psychiatry 73, 565-573 (2013). 
23. Lois, G., Linke, J. \& Wessa, M. Altered functional connectivity between emotional and cognitive resting state networks in euthymic bipolar I disorder patients. PLoS. One. 9, e107829 (2014).

24. Meda, S. A. et al. Differences in resting-state functional magnetic resonance imaging functional network connectivity between schizophrenia and psychotic bipolar probands and their unaffected first-degree relatives. Biol. Psychiatry 71, 881-889 (2012)

25. Teng, $\mathbf{S}$. et al. Classification of bipolar disorder using basal-ganglia-related functional connectivity in the resting state. Conf. Proc. IEEE Eng. Med. Biol. Soc. 2013, 1057-1060 (2013).

26. Altinay, M. I., Hulvershorn, L. A., Karne, H., Beall, E. B. SpringerAmpamp; Anand A. Differential resting-state functional connectivity of striatal subregions in bipolar depression and hypomania. Brain Connect. 6, 255-265 (2016).

27. Mclntyre, R. S., Brecher, M., Paulsson, B., Huizar, K. \& Mullen, J. Quetiapine or haloperidol as monotherapy for bipolar mania-a 12-week, double-blind, randomised, parallel-group, placebo-controlled trial. Eur. Neuropsychopharmacol. 15, 573-585 (2005)

28. Calabrese, J. R. et al. A randomized, double-blind, placebo-controlled trial of quetiapine in the treatment of bipolar I or II depression. Am. J. Psychiatry 162, 1351-1360 (2005)

29. Suppes, T. et al. Maintenance treatment for patients with bipolar I disorder: results from a north american study of quetiapine in combination with lithium or divalproex (trial 127). Am. J. Psychiatry 166, 476-488 (2009).

30. Sachs, G. et al. Quetiapine with lithium or divalproex for the treatment of bipolar mania: a randomized, double-blind, placebo-controlled study. Bipolar Disord. 6, 213-223 (2004)

31. Chai, X. J. et al. Abnormal medial prefrontal cortex resting-state connectivity in bipolar disorder and schizophrenia. Neuropsychopharmacology 36, 2009-2017 (2011).

32. Ongur, D. et al. Default mode network abnormalities in bipolar disorder and schizophrenia. Psychiatry Res. 183, 59-68 (2010).

33. Young, R. C., Biggs, J. T., Ziegler, V. E. \& Meyer, D. A. A rating scale for mania: reliability, validity and sensitivity. Br. J. Psychiatry 133, 429-435 (1978).

34. Montgomery, S. A. \& Asberg, M. A new depression scale designed to be sensitive to change. Br. J. Psychiatry.: J. Ment. Sci. 134, 382-389 (1979).

35. Ventura, J. L. D., Nuechterlein, K. H., Lieberman, R. P., Green, M. \& Shaner, A. Brief Psychiatric Ratings Scale (BPRS) Expanded Version (4.0) scales, anchor points and administration manual. Int. J. Methods Psychiatr. Res. 3, 227-243 (1993).

36. Spearing, M. K., Post, R. M., Leverich, G. S., Brandt, D. \& Nolen, W. Modification of the Clinical Global Impressions (CGI) Scale for use in bipolar illness (BP): the CGI-BP. Psychiatry Res. 73, 159-171 (1997).

37. Brant-Zawadzki, M., Gillan, G. D. \& Nitz, W. R. MP RAGE: a three-dimensional, T1 weighted, gradient-echo sequence--initial experience in the brain. Radiology 182, 769-775 (1992)

38. Fornito, A. et al Functional dysconnectivity of corticostriatal circuitry as a risk phenotype for psychosis. JAMA Psychiatry 70, 1143-1151 (2013)

39. Dandash, O. et al. Altered striatal functional connectivity in subjects with an atrisk mental state for psychosis. Schizophr. Bull. 40, 904-913 (2013).
40. Burton, H., Snyder, A. Z. \& Raichle, M. E. Resting state functional connectivity in early blind humans. Front. Syst. Neurosci. 8, 51 (2014).

41. Ashburner, J. \& Friston, K. J. Unified segmentation. Neuroimage 26, 839-851 (2005).

42. Behzadi, Y., Restom, K., Liau, J. \& Liu, T. T. A component based noise correction method (CompCor) for BOLD and perfusion based fMRI. Neuroimage 37 90-101 (2007)

43. Muschelli, J. et al. Reduction of motion-related artifacts in resting state fMR using aCompCor. Neuroimage 96, 22-35 (2014).

44. Forman, S. D. et al. Improved assessment of significant activation in functional magnetic resonance imaging (fMRI): use of a cluster-size threshold. Magn Reson. Med. 33, 636-647 (1995)

45. Cox, R. W., Chen, G., Glen, D. R., Reynolds, R. C. \& Taylor, P. A. FMRI Clustering in AFNl: False-Positive Rates Redux. Brain Connect 7, 152-71 (2017).

46. Berk, M. et al. Quetiapine v. lithium in the maintenance phase following a first episode of mania: randomised controlled trial. Br. J. Psychiatry 210, 413-421 (2017).

47. Berk, M. et al. Neuroprotection after a first episode of mania: a randomized controlled maintenance trial comparing the effects of lithium and quetiapine on grey and white matter volume. Transl. Psychiatry 7, e1041 (2017).

48. Schutter, D. J. \& van Honk, J. The cerebellum on the rise in human emotion Cerebellum 4, 290-294 (2005).

49. Strick, P. L., Dum, R. P. \& Fiez, J. A. Cerebellum and nonmotor function. Annu. Rev. Neurosci. 32, 413-434 (2009).

50. Schmahmann, J. D. \& Sherman, J. C. The cerebellar cognitive affective syndrome. Brain 121, 561-579 (1998). Pt 4

51. Habel, U., Klein, M., Kellermann, T., Shah, N. J. \& Schneider, F. Same or different? Neural correlates of happy and sad mood in healthy males. Neuroimage $\mathbf{2 6}$ 206-214 (2005).

52. Damasio, A. R. et al. Subcortical and cortical brain activity during the feeling of self-generated emotions. Nat. Neurosci. 3, 1049-1056 (2000).

53. Monkul, E. S. et al. MRI study of the cerebellum in young bipolar patients. Prog. Neuropsychopharmacol. Biol. Psychiatry 32, 613-619 (2008).

54. Berk, M. et al. Neuroprotection after a first episode of mania: a randomized controlled maintenance trial comparing the effects of lithium and quetiapine on grey and white matter volume. Transl. Psychiatry 7, e1011 (2017).

55. Fornito, A., Zalesky, A. \& Breakspear, M. The connectomics of brain disorders Nat. Rev. Neurosci. 16, 159-172 (2015).

56. Kringelbach, M. L. The human orbitofrontal cortex: linking reward to hedonic experience. Nat. Rev. Neurosci. 6, 691-702 (2005).

57. Lui, S. et al. Resting-state brain function in schizophrenia and psychotic bipolar probands and their first-degree relatives. Psychol. Med. 45, 97-108 (2015)

58. Gottesman, I. I. \& Gould, T. D. The endophenotype concept in psychiatry: etymology and strategic intentions. Am. J. Psychiatry 160, 636-645 (2003).

59. Dandash, O. et al. Altered striatal functional connectivity in subjects with an atrisk mental state for psychosis. Schizophr. Bull. 40, 904-913 (2014).

60. Riedel, M. et al. Quetiapine in the treatment of schizophrenia and related disorders. Neuropsychiatr. Dis. Treat. 3, 219-235 (2007). 